\section{Nuclei isolation}

\section{from mouse lung} for single nucleus RNASeq

APR 07, 2020

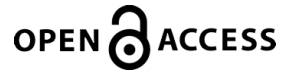

\section{DOI:}

dx.doi.org/10.17504/protocol s.io.bdv2i68e

Protocol Citation: Jeffrey Koenitzer, Ben Humphreys 2020. Nuclei isolation from mouse lung for single nucleus RNASeq. protocols.io https://dx.doi.org/10.17504/p rotocols.io.bdv2i68e

\section{MANUSCRIPT CITATION:} Koenitzer, J. R., Wu, H., Atkinson, J. J., Brody, S. L., Humphreys, B. D. (2020). Single nucleus RNASeq profiling of mouse lung: reduced dissociation bias and improved detection of rare cell types compared with single cell RNASeq. bioRxiv. preprint doi:

https://doi.org/10.1101/2020. 03.06.981407

License: This is an open access protocol distributed under the terms of the Creative Commons Attribution License, which permits unrestricted use, distribution, and reproduction in any medium, provided the original author and source are credited

Protocol status: Working We use this protocol and it's working

\section{(3) Nuclei isolation from mouse lung for single nucleus RNASeq}

Ben

Jeffrey Koenitzer ${ }^{1}$, Humphreys ${ }^{2,3}$

${ }^{1}$ Division of Pulmonary and Critical Care, Department of Medicine, Washington University in St. Louis School of Medicine, USA;

${ }^{2}$ Division of Nephrology, Department of Medicine, Washington University in St. Louis School of Medicine, USA;

${ }^{3}$ Department of Developmental Biology, Washington University in St. Louis School of Medicine, USA

Human Cell Atlas Method Development Community<smiles>O=C(CBr)C1CCCCCCCC1</smiles>

\section{ABSTRACT}

This protocol is for nuclei isolation from mouse lung for single nucleus RNASeq.

It is adapted directly from Joshi et al., with adjustments to RNase inhibitor concentrations and removal of FACS sorting steps.

\section{CITATION}

Nikita Joshi, Alexander Misharin. Single-nucleus isolation from frozen human lung tissue for single-nucleus RNA-seq.

LINK

dx.doi.org/10.17504/protocols.io.zu8f6zw

\footnotetext{
ATTACHMENTS

Protocol for nuclei isolation from mouse lung for single nucleus RNASeq.pdf

step-by-step_Lung nuclear isolation protocol v1.pdf

\section{MATERIALS}

MATERIALS
} 
Created: Mar 18, 2020

Last Modified: Apr 07, 2020

PROTOCOL integer ID: 34458

Keywords: nuclei isolation, mouse, mouse lung, RNASeq single nucleus RNASeq
88 RNaseZap® Thermo Scientific Catalog \#AM9780

88 cOmplete ULTRA Tablets, Mini, EDTA-free, EASYpack Roche Catalog \#05 89279 001

88 RNasin Plus Ribonuclease Inhibitors Promega Catalog \#N2615

88 SUPERaseIN RNase Inhibitor Thermo Fisher Scientific Catalog \#AM2696

88 RNase free H2O Thermo Scientific Catalog \#AM9938

89 Albumin, Bovine Serum, 10\% Aqueous Solution, Nuclease-Free Millipore Sigma Catalog \#126615-25ML

Gibco $^{\text {Th }}$ DPBS no calcium no magnesium Thermo Fisher Scientific Catalog 88 \#14190144

$8 \%$

Nuclei Isolation Kit: Nuclei EZ Prep Sigma Aldrich Catalog \#NUC101

Note

The Nuclei Isolation Kit contains the Nuclei EZ Lysis Buffer that is required for the praparation of Lysis Buffer and cOmplete stock (10x).

\section{Storage Conditions}

\begin{tabular}{|l|l|}
\hline $\begin{array}{l}\text { Mater } \\
\text { ial }\end{array}$ & $\begin{array}{l}\text { Stora } \\
\text { ge }\end{array}$ \\
\hline 1x & $4^{\circ} \mathrm{C}$ \\
DPBS & ${ }^{\circ}$ \\
\hline Nuclei & \\
EZ \\
Lysis \\
Buffer & \\
(from & $4{ }^{\circ} \mathrm{C}$ \\
kit & \\
NUC- & \\
$101)$ & \\
\hline cOmp & \\
lete & \\
ULTR & \\
A & \\
Tablet & \\
S, & $4{ }^{\circ} \mathrm{C}$ \\
Mini, & \\
EDTA- & \\
free, & \\
EASY & \\
pack &
\end{tabular}




\begin{tabular}{|l|l|}
\hline RNasi & \\
n Plus & \\
Ribon & \\
uclea & $-20{ }^{\circ} \mathrm{C}$ \\
se & \\
Inhibit & \\
ors & \\
\hline SUPE & \\
Rasel & \\
N & \\
RNas & $-20{ }^{\circ} \mathrm{C}$ \\
e & \\
Inhibit & \\
or & \\
\hline RNas & \\
e free & RT \\
H2O & \\
\hline RNas & RT \\
eZap & \\
\hline Bovin & \\
e & \\
seru & \\
m & \\
album & \\
in, & $-20{ }^{\circ} \mathrm{C}$ \\
$10 \%$ & \\
soluti & \\
on, & \\
nucle & \\
ase & \\
free & \\
\hline
\end{tabular}

\section{Equipment}

\section{gentleMACS ${ }^{\mathrm{TM}} \mathrm{C}$ Tubes}

Tissue dissociators and tubes

gentleMACS ${ }^{\text {TM }}$

https://www.miltenyibiotec.com/AD-en/products/macs-sample-

preparation/tissue-dissociators-and-tubes/c-tubes/gentlemacs-c-tubes.html 


\section{Equipment}

\section{gentleMACS ${ }^{\mathrm{TM}}$ Dissociator}

Tissue Dissociator

MACS

https://www.miltenyibiotec.com/US-en/products/macs-samplepreparation/tissue-dissociators-and-tubes/gentlemacsdissociator/gentlemacstm-dissociator.html

\section{Equipment}

pluriStrainer ${ }^{\circledR} 40 \mu \mathrm{m}$

Cell Strainer

pluriSelect

\section{Equipment}

pluriStrainer ${ }^{\circledR} 5 \mu \mathrm{m}$

Cell Strainer

pluriSelect

https://www.pluriselect.com/eu/pluristrainerr-5-m-cell- 


\section{Equipment}

C-Chip $^{\text {TM }}$ Disposable Hemacytometers (Fuchs Rosenthal)

Counting Chamber

INCYTO

DHCF015

https://us.vwr.com/store/product/4618278/incyto-c-chiptm-disposablehemacytometers-skc

\section{Equipment}

Falcon $^{\mathrm{TM}} 15 \mathrm{~mL}$ Polystyrene Conical Centrifuge Tubes

Centrifuge Tubes

Falcon $^{\text {TM }}$

https://www.fishersci.com/shop/products/falcon-15ml-conical-centrifuge- LINK tubes-5/p-193301 


\section{Equipment}

\section{Ambion ${ }^{\circledR}$ RNase-free $50 \mathrm{ml}$ Conical Tubes}

Centrifuge Tubes

Ambion ${ }^{\circledR}$

https://www.thermofisher.com/order/catalog/product/AM12502\#/AM1250 LINK 2

\section{Equipment}

TPP 60 mm Tissue Culture Dishes

Tissue Culture Dish

TPP

TP93060

https://midsci.com/item/ASTPPDISH/TPP-Dishes/

\section{SAFETY WARNINGS}

(1) Please see SDS (Safety Data Sheet) for hazards and safety warnings. 


\section{BEFORE START INSTRUCTIONS}

\section{Prepare Working Solutions}

Complete stock (10x)

- I $_{\text {buffer }}^{1 \mathrm{~mL} \text { Nuclei EZ Prep lysis }}$

- I 1 tablet cOmplete ULTRA tablets

Lysis Buffer $-2 \mathrm{ml}$ per $<6 \mathrm{~mm}^{3}$ tissue

- I $200 \mu \mathrm{L}$ cOmplete stock

ـ $1.775 \mathrm{~mL}$ Nuclei EZ Prep lysis

buffer

- $12.5 \mu \mathrm{L}$ RNasin

- $\triangle$ Plus

- \ $12.5 \mu \mathrm{L}$ SUPERaselN

Wash Buffer - $4 \mathrm{ml}$ per $<6 \mathrm{~mm}^{3}$ tissue

- I $3.575 \mathrm{~mL}$

- I $400 \mu \mathrm{L} \mathrm{10 \%}$

- I BSA

- $\underset{\text { Plus }}{12.5 \mu \mathrm{L} \text { RNasin }}$

- \ $12.5 \mu \mathrm{L}$ SUPERaseIN

Resuspension Buffer - $1 \mathrm{ml}$

- $80.977 \mathrm{~mL}$

dPBS

- I $10 \mu \mathrm{L} 10 \%$

BSA

I $6.25 \mu \mathrm{L}$ RNasin

8 Plus

- I $6.25 \mu \mathrm{L}$ SUPERaseIN

1 Pre-cool all instruments (including centrifuges), buffers, and tubes.

Note

Note: All steps are performed $8^{\circ}$ On ice or in cold room $\left(8^{\circ} 4{ }^{\circ} \mathrm{C}\right)$ to minimize RNA degradation. 
2 Remove mouse lung sample from $-80^{\circ} \mathrm{C}$ freezer and trim a $\sim 6 \mathrm{~mm}^{3}$ piece.

3 Thaw on small plastic weighing boat until able to insert $26 \mathrm{G}$ needle to tissue, then inject ice cold $1 \mathrm{~mL}$ Lysis to 'inflate' the tissue. Add the remaining Lysis Buffer and chop to the
Buffer smallest pieces possible with scissors (60 s).

$4 \quad$ Transfer the minced tissue and buffer to a GentleMacs $C$ tube.

5 Close, invert, and transfer directly to MACS Tissue dissociator.

6 Run $m \_l u n g \_01$ program and $m \_/ u n g \_02$ program in sequence, stopping the latter after (3) 00:00:20 


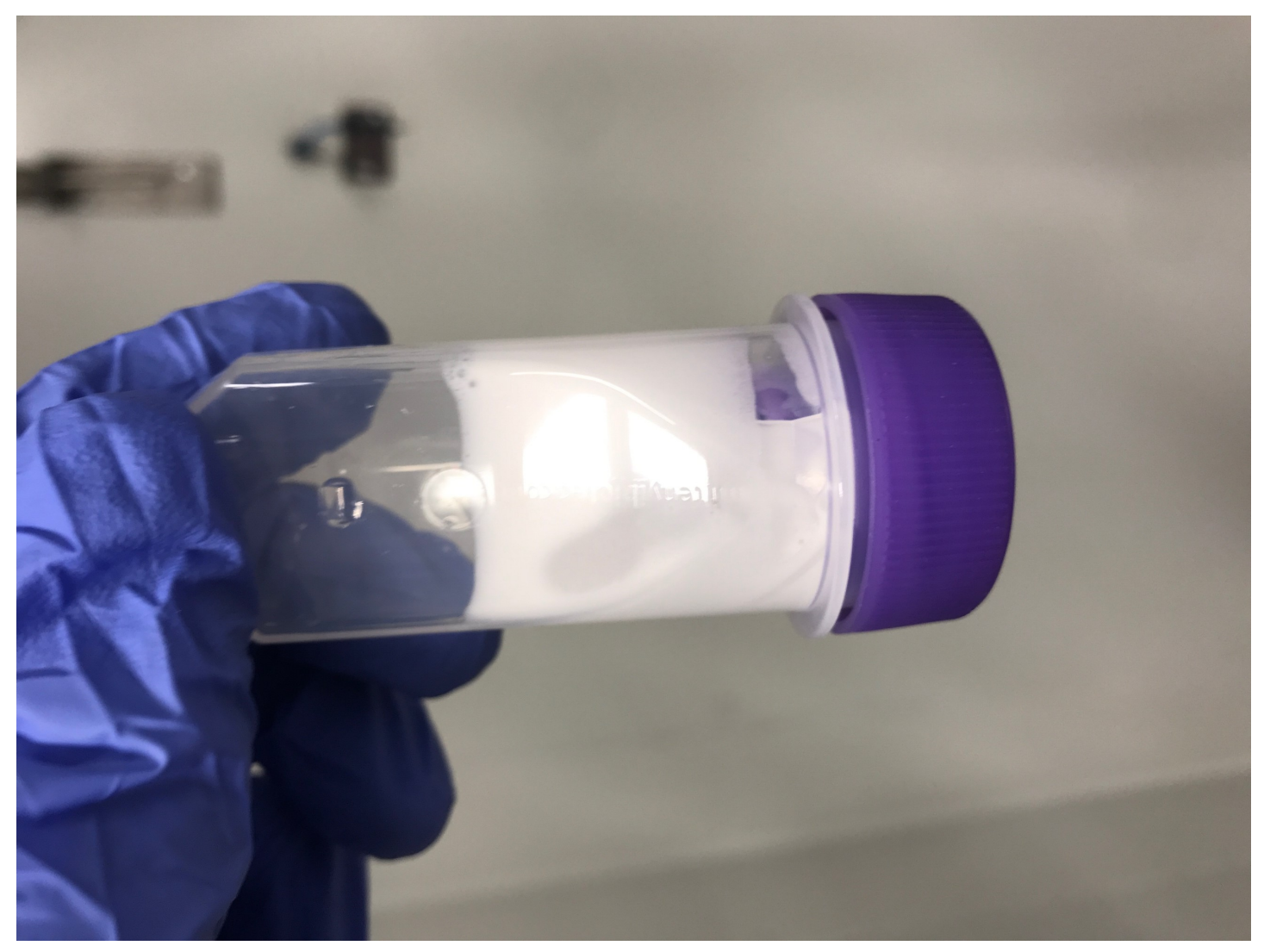

Foam after GentleMacs

$7 \quad$ Place tube $\AA^{\circ}$ On ice

$8 \quad$ Reduce foam by centrifugation $\left(\because: \begin{array}{l}750 \times \mathrm{g}, \\ 00: 01: 00\end{array}\right)$. 


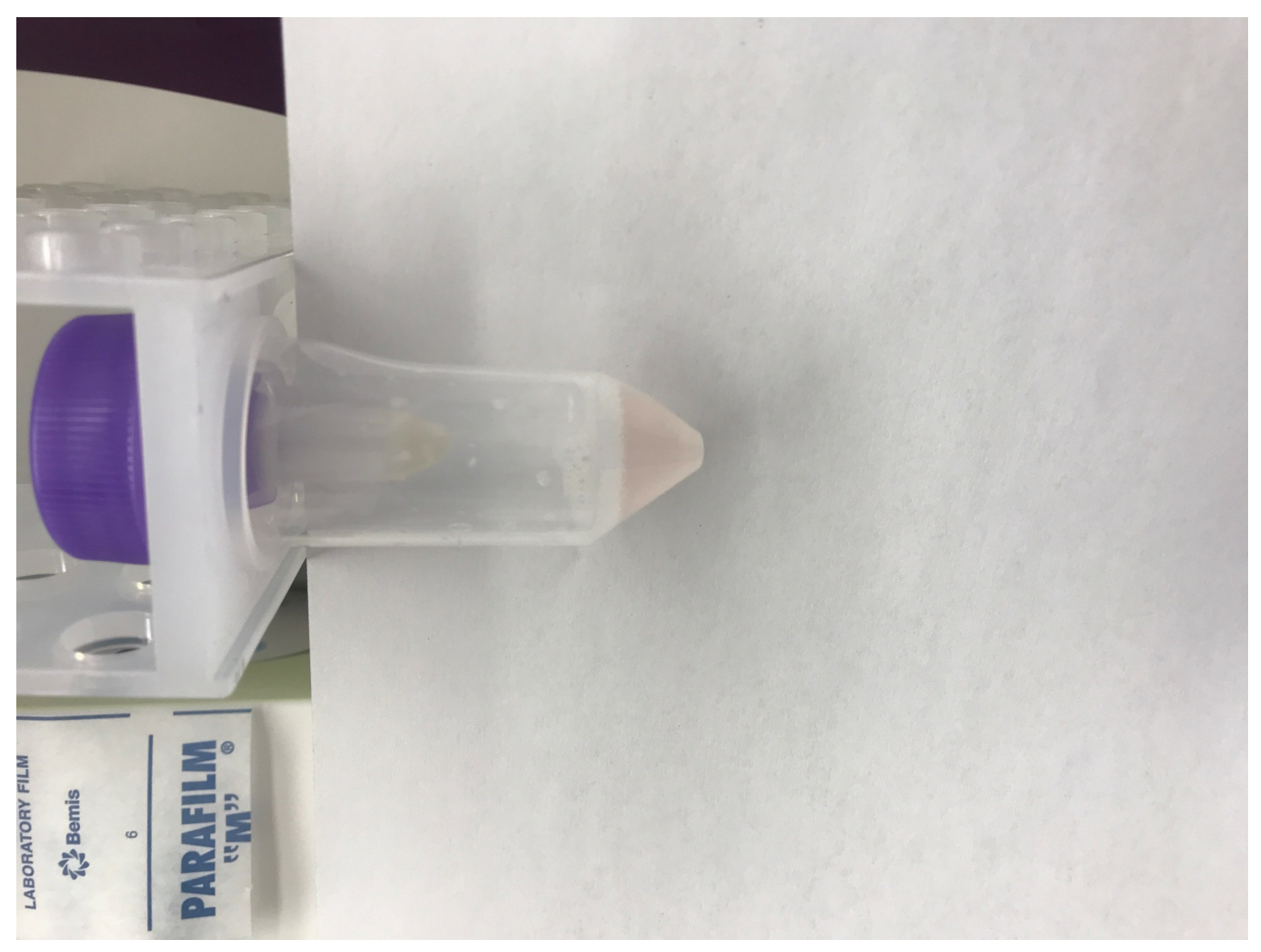

Foam after centrifugation

9 Using a wide bore tip, pipette up and down to recover any pelleted material and pass lysate through $40 \mu \mathrm{m}$ filter to $50 \mathrm{ml}$ conical tube.

10 Wash strainer with $\Delta_{\text {Buffer }}^{4 \mathrm{~mL} \text { Wash }}$

11 Pass suspension through a $5 \mu \mathrm{m}$ strainer into $50 \mathrm{ml}$ conical tube.

$12 \quad$ Centrifuge at $\because: \begin{aligned} & 600 \times \mathrm{g}, 4^{\circ} \mathrm{C}, \\ & 00: 05: 00\end{aligned}$ 
13

Resuspend in $\triangle \Delta \begin{aligned} & 500 \mu \mathrm{L} \text { Resuspension } \\ & \text { Buffer }\end{aligned}$

14 Count nuclei by hemocytometer and dilute to desired concentration (e.g. 10,000 per $\mu \mathrm{l}$ ).

Sote

Note: it is easier to resuspend in lower volumes and dilute than to concentrate nuclei via further centrifugation.
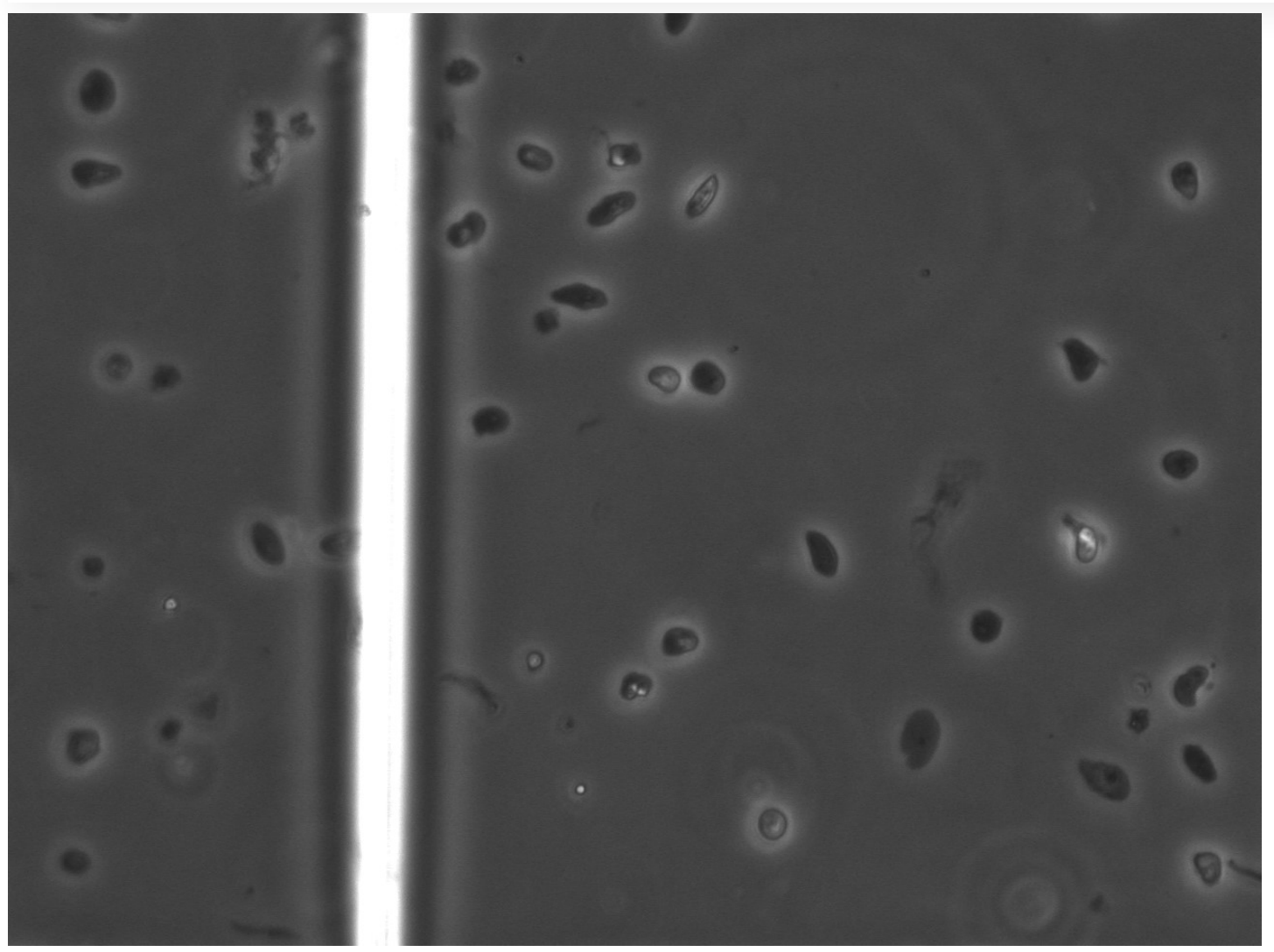

40x Nuclear suspension with scant debris

15 Proceed to 10x Chromium. 
